
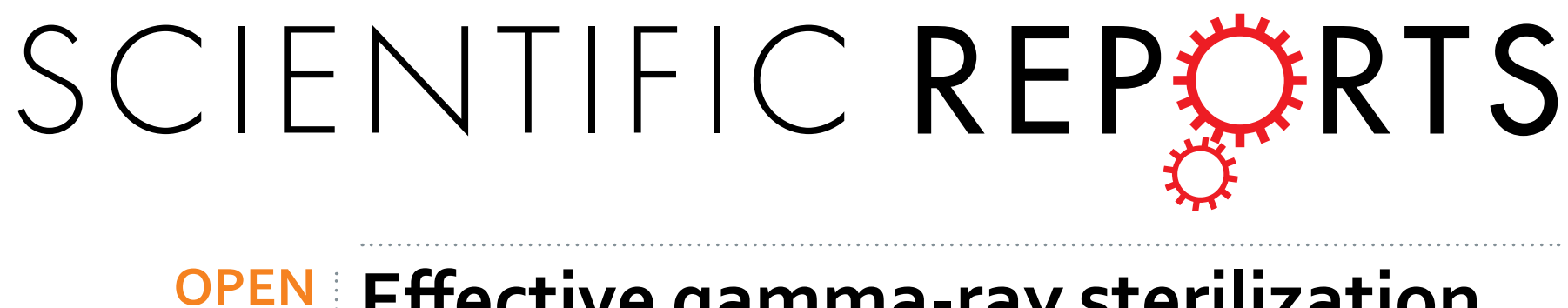

\title{
Effective gamma-ray sterilization and characterization of conductive polypyrrole biomaterials
}

Received: 27 November 2017

Accepted: 15 February 2018

Published online: 27 February 2018
Semin Kim ${ }^{1}$, Jin-Oh Jeong ${ }^{1,2}$, Sanghun Lee ${ }^{3}$, Jong-Seok Park², Hui-Jeong Gwon ${ }^{2}$, Sung In Jeong ${ }^{2}$, John George Hardy $\mathbb{1 D}^{4,5}$, Youn-Mook Lim² \& Jae Young Lee $\mathbb{D}^{1,6}$

Conductive polymers, including polypyrrole (PPy), have been extensively explored to fabricate electrically conductive biomaterials for bioelectrodes and tissue engineering scaffolds. For their in vivo uses, a sterilization method without severe impairment of original material properties and performance is necessary. Gamma-ray radiation has been commonly applied for sterilization of medical products because of its simple and uniform sterilization without heat generation. Herein we describe the first study on gamma-ray sterilization of PPy bioelectrodes and its effects on their characteristics. We irradiated PPy bioelectrodes with different doses (0-75 kGy) of gamma-rays. Gamma-ray irradiation of the PPy ( $\gamma$-PPy) increased the oxygenation and hydrophilicity of the surfaces. Interestingly, gamma-ray irradiation did not alter the electrical impedances and conductivities of the PPy substrates. Additionally, $\gamma$-PPy prepared with various dopants (e.g., para-toluene sulfonate, polystyrene sulfonate, and chlorine) showed the electrochemical properties similar to the non-irradiated control. Gamma-ray irradiation at doses of $\geq \mathbf{1 5} \mathrm{kGy}$ was required for effective sterilization as evidenced by complete eradication of gram positive and negative bacteria. $\gamma$-PPy substrates also showed cytocompatibility similar to untreated control PPy, indicating no substantial alteration of cytocompatibility. In conclusion, gamma ray sterilization is a viable method of sterilization of conducting polymer-based biomaterials for biomedical applications.

Conducting polymers (CPs) have attracted attention from scientists and engineers due to their high electrical conductivity, simple synthesis, and excellent biocompatibility ${ }^{1,2}$. CPs have also been extensively explored to fabricate electrically conductive biomaterials, which allow for efficient delivery of electrical signals with low electrochemical impedance, high charge injection capability, and biocompatibility ${ }^{3,4}$, for bioelectrodes and tissue engineering scaffolds applications ${ }^{1,5,6}$. Commonly used CPs include poly(3,4-ethylenedioxythiophene) (PEDOT), polyaniline (PANi), polythiophene (PT), and polypyrrole (PPy). CPs are recognized as promising materials for the development of neural prosthetics ${ }^{7-10}$, cochlear implants ${ }^{5,11}$, drug delivery devices ${ }^{12,13}$, bioactuators ${ }^{14,15}$, and biosensors ${ }^{4,16,17}$.

To clinically translate CP-based biomaterials including bioelectrodes for implantation in the body, effective sterilization methodologies need to be established which eradicate bacterial infection and ensure their biocompatibility. Sterilization of CP-based biomaterials for in vivo uses is challenging because it may lead to the deterioration of the CP's inherent electrical and biochemical properties, and there is a lack of systematic studies validating the effects of sterilization methods on the properties of such CP-based biomaterials. Conventional sterilization methods, such as steam, ultraviolet radiation (UV), ethylene oxide (EO), and gamma-irradiation sterilization, can be employed for $\mathrm{CPs}^{18,19}$. Among them, sterilization by gamma-ray irradiation offers several

\footnotetext{
${ }^{1}$ School of Materials Science and Engineering, Gwangju Institute of Science and Technology, Gwangju, 61005, Republic of Korea. ${ }^{2}$ Research Division for Industry \& Environment, Advanced Radiation Technology Institute, Korea Atomic Energy Research Institute (KAERI), 29 Gumgugil, Jeongeup, 56212, Republic of Korea. ${ }^{3}$ Materials Science and Engineering Concentration, GIST College, Gwangju Institute of Science and Technology, Gwangju, 61005, Republic of Korea. ${ }^{4}$ Department of Chemistry, Lancaster University, Lancaster, Lancashire, LA1 4YB, United Kingdom. ${ }^{5}$ Materials Science Institute, Lancaster University, Lancaster, Lancashire, LA1 4YB, United Kingdom. ${ }^{6}$ Department of Biomedical Science and Engineering, Gwangju Institute of Science and Technology, 500-712 Republic of Korea, Gwangju, 61005, Republic of Korea. Semin Kim and Jin-Oh Jeong contributed equally to this work. Correspondence and requests for materials should be addressed to Y.-M.L. (email: jaeyounglee@gist.ac.kr) or J.Y.L. (email: ymlim71@kaeri.re.kr)
} 
(a)

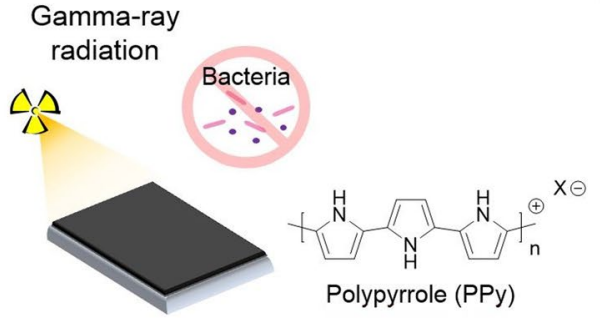

(b)

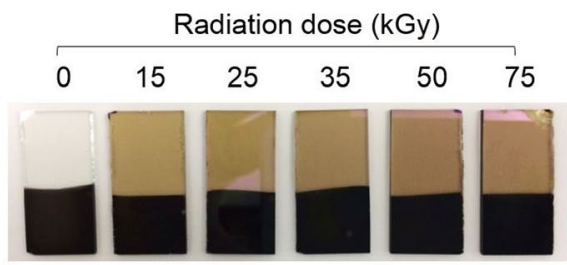

Figure 1. (a) A schematic illustration of gamma-ray sterilization of PPy biomaterials. (b) Photographs of the PPy-deposited ITO electrodes after exposure to different doses of gamma-rays.

important advantages, including: gamma-rays can easily and uniformly reach all parts of the object to be sterilized; sterilization can be performed with different doses for various materials (e.g., heat-sensitive materials) in various environments (e.g., at low temperatures in gas, solid, or liquid states $)^{20-22}$. However, irradiation with high-energy gamma-ray can cause various chemical reactions in polymers, including polymer chain scission, crosslinking, and degradation ${ }^{23-26}$. An excessive dose of gamma-ray can lead to the alteration of material's chemical, electrical, mechanical and biological properties. The focus of this study is to understand the effects of gamma-ray irradiation on both sterilization effectiveness and materials properties of PPy bioelectrodes, which are of critical importance for their eventual translation.

In this study, we synthesized para-toluene sulfonate (pTS)-doped PPy (PPy/pTS) coated electrodes and exposed them to various doses of gamma-rays to study the suitability of gamma-ray radiation for the sterilization of CP-based bioelectrodes (Fig. 1). PPy has been widely studied for biomedical applications (e.g., electrodes, biosensors, and tissue engineering scaffolds) due to its facile synthesis, high electrical conductivity, excellent stability, and good biocompatibility $27-31$. We carefully characterized the chemical, electrochemical, and biological properties of the irradiated PPy/pTS ( $\gamma$-PPy) electrodes using a variety of methods. In addition, the effectiveness of sterilization via gamma-ray irradiation was assessed with gram positive and gram negative bacteria. Finally, the cytocompatibility of the $\gamma$-PPy substrates was studied using various types of cells (e.g., neuronal cells, myoblasts, and fibroblasts).

\section{Results}

Electrochemical fabrication of PPy electrodes and gamma-ray irradiation. A PPy $/ \mathrm{pTS}$ was electrochemically polymerized on ITO or gold electrodes (Supplementary Fig. S1), followed by exposure to gamma-rays. The PPy/PSS substrates irradiated with different radiation doses $(0,15,25,35,50$, and $75 \mathrm{kGy})$ were denoted as $\gamma$-PPy $x$, in which $x$ indicates a dose (kGy). After gamma ray irradiation, the ITO-glass changed color from transparent to light brown (Fig. 1b). The various $\gamma$-PPy samples were characterized via a variety of techniques as shown in the following results.

Characterization of gamma-irradiated PPy electrodes. The surface morphologies of the PPy and $\gamma$-PPy were analyzed by AFM. As shown in Fig. 2, both PPy and various $\gamma$-PPy exhibit similar surface features with numerous spherical nodules with grains of similar sizes $(100-200 \mathrm{~nm})$ analogous to those reported by other researchers ${ }^{32}$. The surface roughness was not significantly different between PPy and $\gamma$-PPy. Furthermore, SEM images of PPy and $\gamma$-PPy confirm that gamma-ray irradiation does not influence the morphology of the PPy surfaces (Supplementary information Fig. S2).

Gamma-ray radiation increased the hydrophilicity of the PPy samples (Fig. 3). Hydrophilicity of the PPy surfaces increased with the increased doses of gamma-ray from 15 to $75 \mathrm{kGy}$. Non-irradiated PPy had a water contact angle of $58.04 \pm 1.92^{\circ}$, whereas those of $\gamma$-PPy irradiated with $15,25,35,50$, and $75 \mathrm{kGy}$ were $29.33 \pm 1.02$, $28.06 \pm 0.62,25.74 \pm 1.15,21.90 \pm 0.73$, and $19.35 \pm 1.26^{\circ}$, respectively. Compared to the PPy, the $\gamma$-PPy 75 had approximately $40^{\circ}$ smaller contact angles.

The chemical compositions of $\gamma$-PPy surfaces were analyzed by XPS and compared with that of non-irradiated PPy (Fig. 4). High-resolution $\mathrm{C}_{1 \mathrm{~s}}$ spectra of the PPy and $\gamma$-PPy films were deconvoluted into four peaks, which included $284.6(\underline{\mathrm{C}}-\mathrm{C} / \underline{\mathrm{C}}-\mathrm{H}), 285.5(\underline{\mathrm{C}}-\mathrm{N}), 286.8(\underline{\mathrm{C}}-\mathrm{O})$, and $288.4 \mathrm{eV}(\underline{\mathrm{C}}=\mathrm{O})$, according to the literatures ${ }^{9}$. With an increase in gamma-ray dose, the intensities of the peaks at $286.9 \mathrm{eV}(\underline{\mathrm{C}}-\mathrm{O})$ and $288.4 \mathrm{eV}(\underline{\mathrm{C}}=\mathrm{O})$ increased. The sum of relative intensities of these oxygenated carbons $(\mathrm{C}-\mathrm{O}$ and $\mathrm{C}=\mathrm{O})$ were $14.7 \%$ for PPy, whereas $\gamma$-PPy-75 had $21.3 \%$ of these oxygenated carbon peaks, respectively. Consequently, gamma ray irradiation caused more oxygenation on the PPy surfaces. Ozone and oxygen radicals would be generated during gamma-ray irradiation which reacts with the oxygen and/or water molecules in the air, which oxidises the PPy, which is supported by surface elemental analysis indicating more oxygen atoms on the PPy electrodes irradiated with the higher doses.

The adhesion of PPy coatings on the electrodes after gamma-ray irradiation was examined by using the Scotch tape detachment test (Supplementary information Fig. S3). The pristine PPy coating was easily detached from the ITO electrode by scotch tape. For the $\gamma$-PPy, the PPy coating was stronger with higher doses of gamma-ray, implying the contribution of gamma-ray for the formation of stronger intermolecular interactions between the electrode surfaces and PPy films. 
(a)
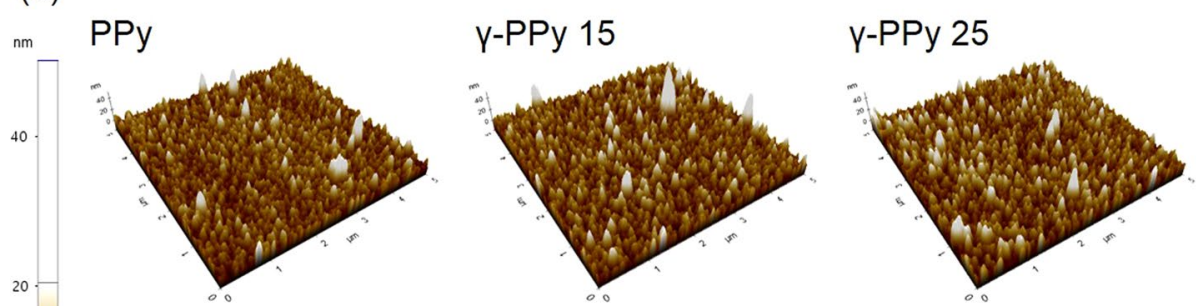

(b)
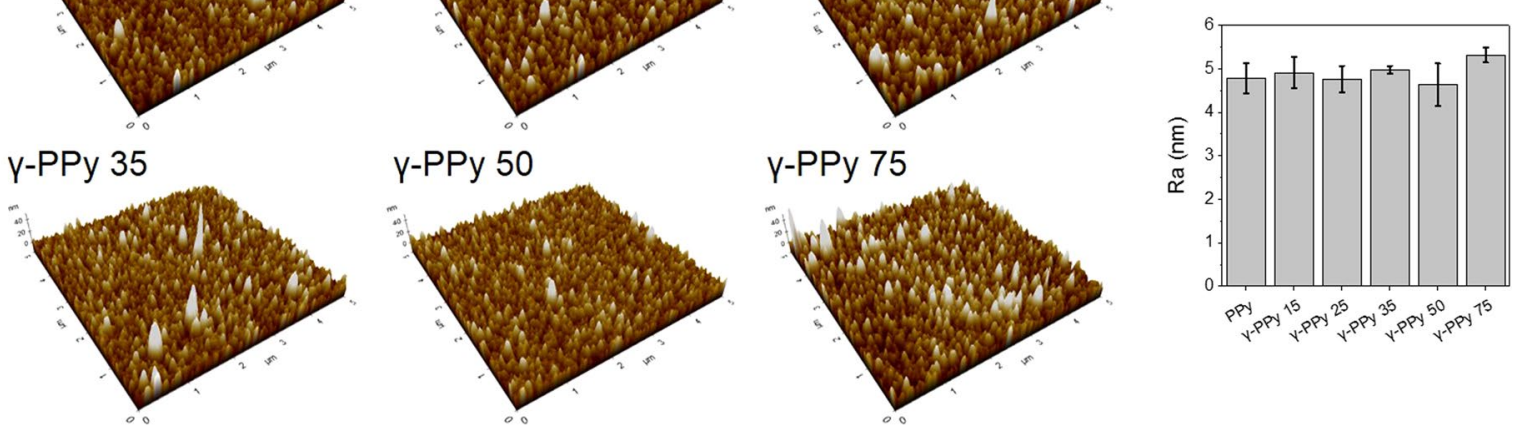

Figure 2. (a) Atomic force micrographs of PPy and $\gamma$-PPy samples irradiated with different doses of gammaray. (b) Average roughness (root mean square) of PPy and $\gamma$-PPy samples.

(a)

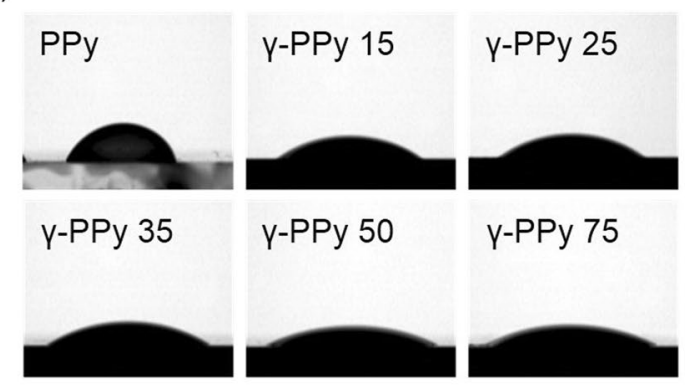

(b)

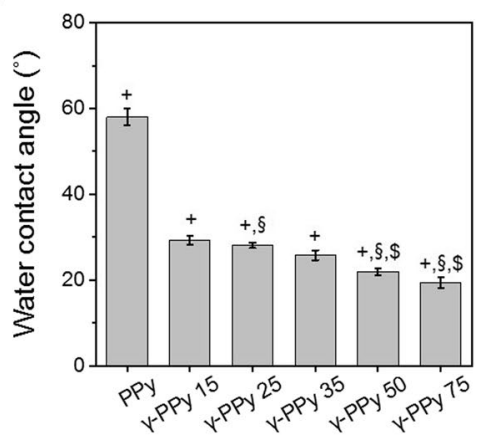

Figure 3. Water contact angle measurement. (a) Photographs of water droplets on PPy and $\gamma$-PPy electrodes. (b) A plot of water contact angles $\left(n=3,{ }^{+} \mathrm{p}<0.05\right.$ versus PPy, ${ }^{{ }_{\mathrm{p}}} \mathrm{p}<0.05$ versus $\gamma$-PPy $25,{ }^{\$} \mathrm{p}<0.05$ versus $\gamma$-PPy 25.

Electrochemical and electrical properties of the $\gamma$-PPy. Electrochemical impedance spectroscopy (EIS) was employed to evaluate the electrical/electrochemical properties of the PPy electrodes before and after gamma-ray irradiation. Possible alteration of the electrical properties of electrodes after sterilization should be carefully examined to ensure their inherent electrode performances. The impedances of $\gamma$-PPy electrodes were similar to those of non-irradiated PPy over the frequencies tested (Fig. 5a,b). These results suggest that gamma-ray irradiation with 15-75 kGy doses did not impair the electrode's original electrochemical properties. For comparison, we autoclaved the PPy electrodes and measured the EIS. We tested 10 autoclaved samples (Supporting Information Fig. S4), finding that the averages of the impedances of all the autoclaved PPy electrodes (noted as 'Autoclave $b$ ') showed higher impedances than pristine PPy electrodes or gamma irradiated PPy electrodes. Five of the 10 autoclaved electrodes showed relatively good conductance, which was noted as 'Autoclave $a$ ' in the Fig. 5b. The reason of high increases in impedances of autoclaved PPy electrodes (5 out of 10) was attributed to local detachment of the PPy films from the ITO electrodes (Supporting Information Fig. S5), which occurs during autoclaving the samples due to differences in the thermal expansion of PPy and ITO. In addition, cyclic voltammetry indicates that the redox peaks, peak potential separation and capacitance of $\gamma$-PPy electrodes were not significantly changed by gamma-ray radiation (Fig. 5c, Supporting Information Fig. S6). The conductivity of the $\gamma$-PPy was not significantly different from the non-irradiated PPy, suggesting no impairment of inherent molecular structures or electronic properties of PPy after gamma-ray irradiation (Fig. 5d). UV-vis spectroscopy was employed to examine the changes in electronic energy states of $\gamma$-PPy after gamma-ray radiation (Fig. 5e). No significant shift of the peaks corresponding polaron-biopolaron transition at $500-510 \mathrm{~nm}$ was observed for the $\gamma$-PPy samples. These peaks are indicative of lengths of PPy chains and conduction bands ${ }^{33}$, which suggests that the electronic states and conjugation structures of PPy were stable to the doses of gamma-ray irradiation to which they were exposed.

Moreover, we explored the possible effects of dopants on changes in electrochemical properties of PPy after gamma-ray irradiation (Fig. 6). Three different dopants were used for the preparation of PPy-electrodes, which included PPy/pTS, PPy/PSS, and PPy/Cl. The radiation did not significantly affect impedances of PPy/PSS at all frequency ranges, which was similar to the results of pTS-doped PPy (PPy/pTS). For $\gamma-\mathrm{PPy} / \mathrm{Cl}$, impedances 

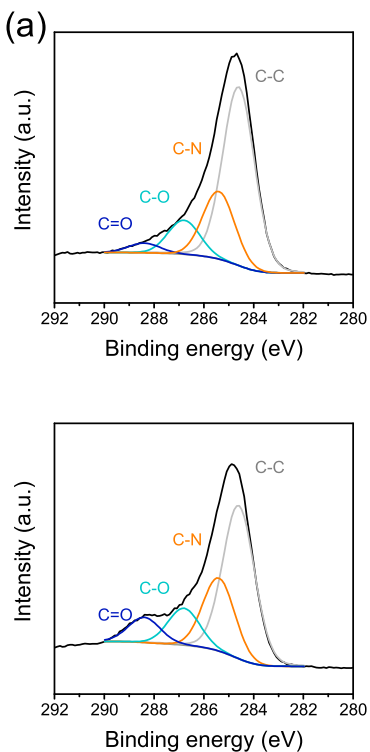
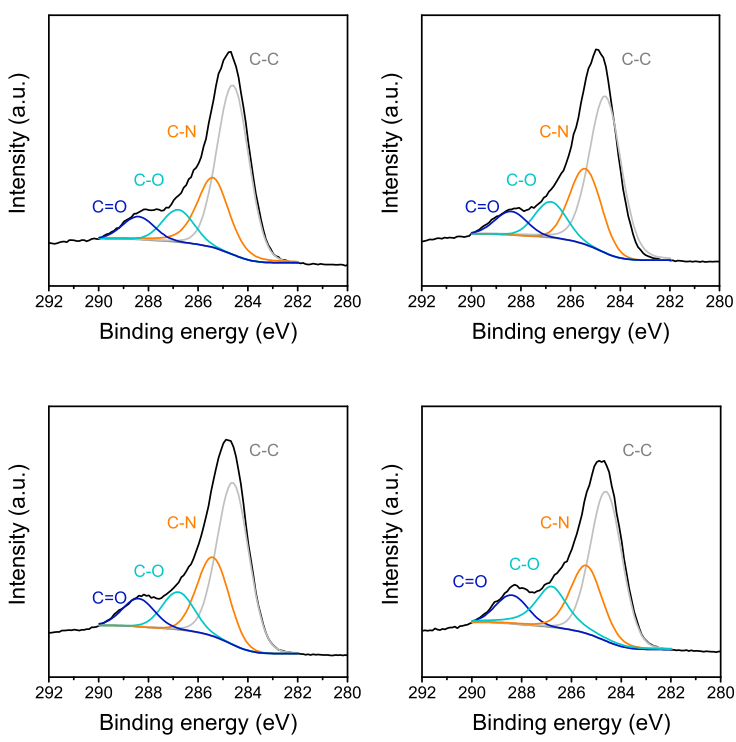

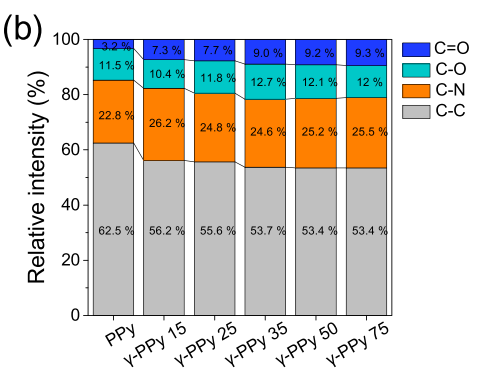

(c)

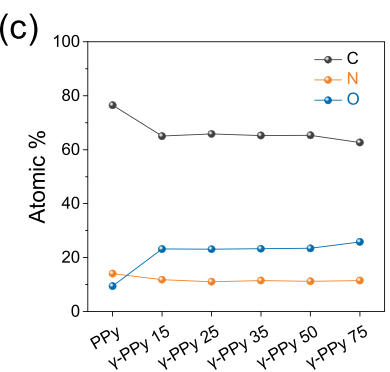

Figure 4. XPS spectra of PPy and $\gamma$-PPy surfaces. (a) High-resolution $\mathrm{C}_{1 \mathrm{~s}}$ spectra with deconvoluted peaks from the PPy and $\gamma$-PPy. (b) Relative intensities of carbon peaks in the spectra. (c) Relative composition of carbon, nitrogen, and oxygen atoms on the surfaces.

(a)

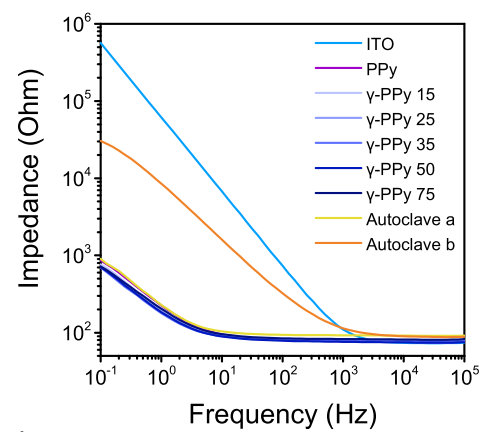

(c)

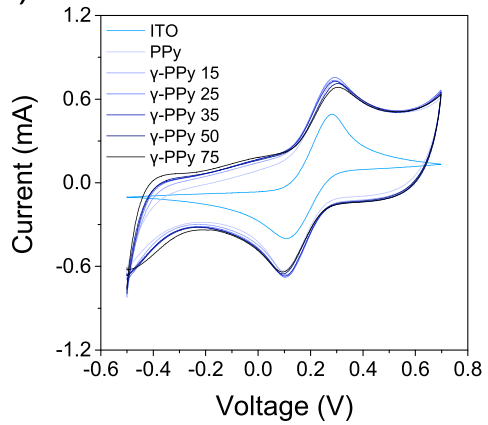

(b)

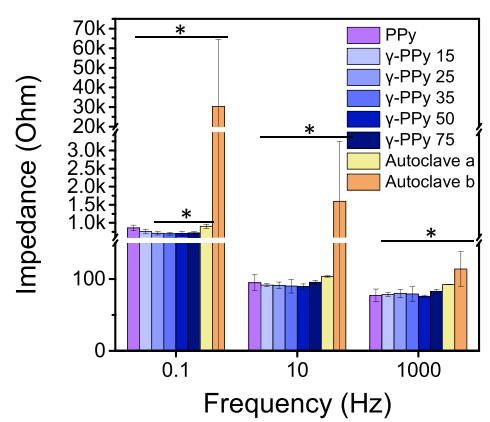

(d)

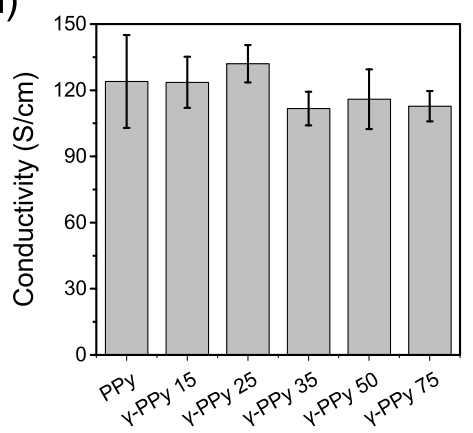

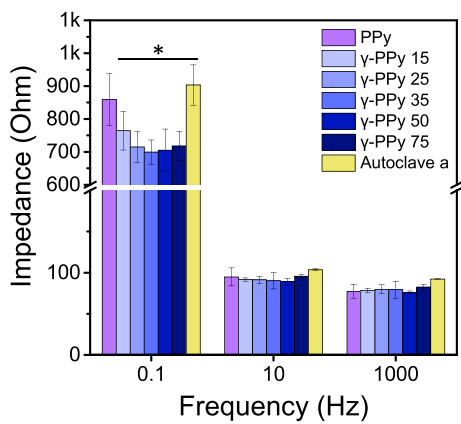

(e)

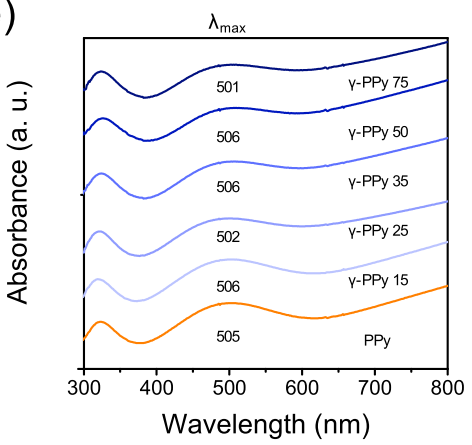

Figure 5. Characterization of electrochemical and electrical properties of the electrodes. (a) Bode plots and (b) Impedances at $0.1,1$, and $10^{3} \mathrm{~Hz}$ of various electrodes. Ten PPy electrodes were autoclaved. Among them, five electrodes showing the lowest impedances were selected and noted as 'Autoclave $a$ ' and all the 10 autoclaved electrodes were noted as 'Autoclave b' in the plots. (c) Cyclic voltammograms of the electrodes in a presence of $5 \mathrm{mM}\left[\mathrm{Fe}(\mathrm{CN})_{6}\right]^{3-/ 4-}$ in $0.1 \mathrm{M} \mathrm{KCl}$ solution at a $0.1 \mathrm{~V} / \mathrm{s} \mathrm{scan}$ rate. (d) Conductivity of PPy and $\gamma-\mathrm{PPy}$. (e) UV/vis spectra of PPy and $\gamma$-PPy electrodes.

slightly increased after irradiation; however, these differences were not significant. The results imply that gamma-ray irradiation can be employed for different PPy-based biomaterials doped with various dopants with minimal deterioration of the electrochemical characteristics of the PPy electrodes. 
(a)
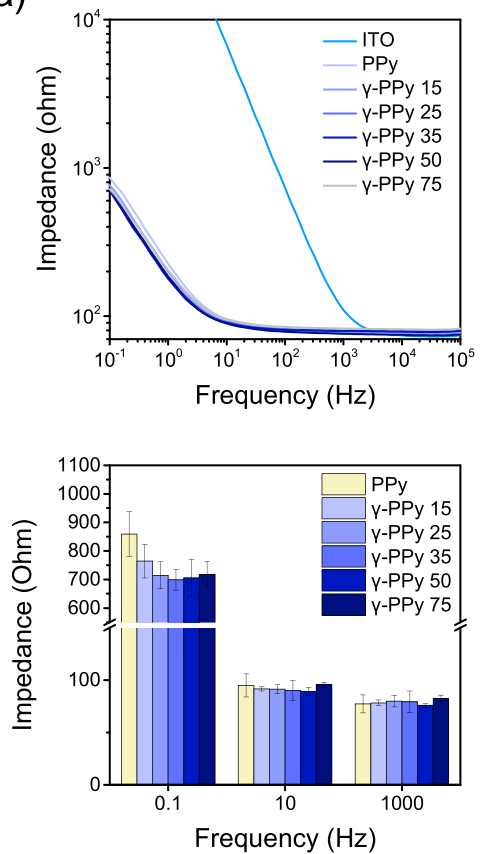

(b)
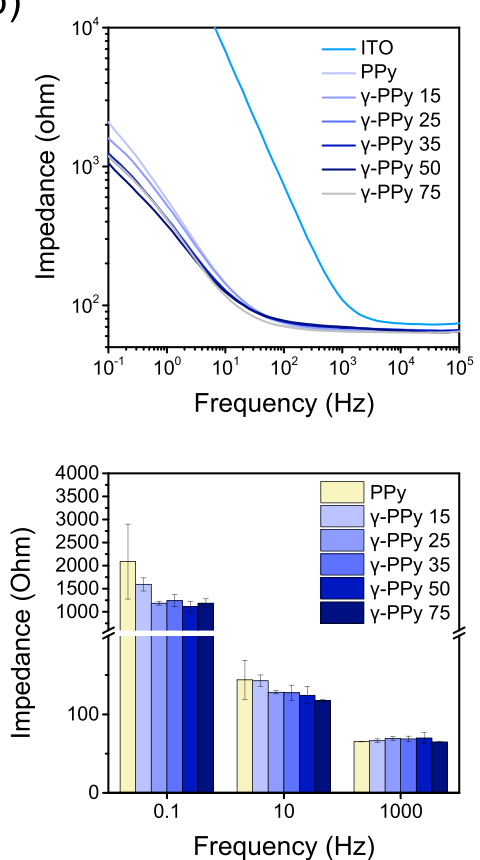

(c)
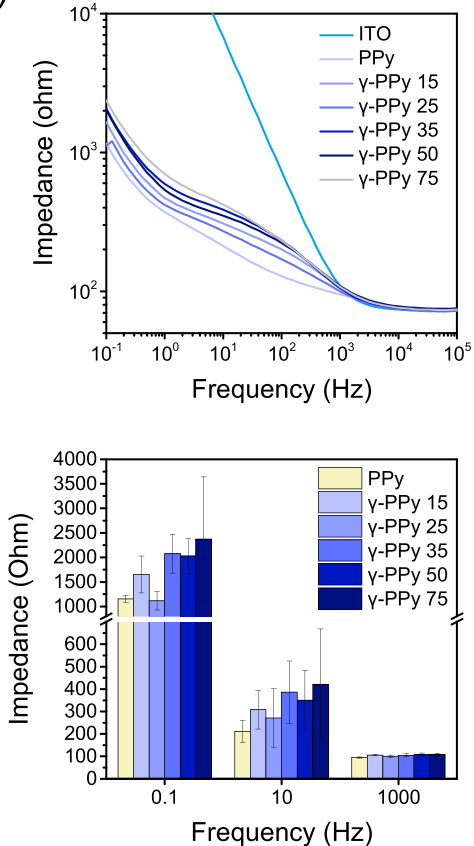

Figure 6. Electrochemical properties of PPy electrodes prepared with different dopants, (a) PPy/pTS, (b) PPy/ PSS, (c) PPy/Cl, after the gamma-ray irradiation with different doses.
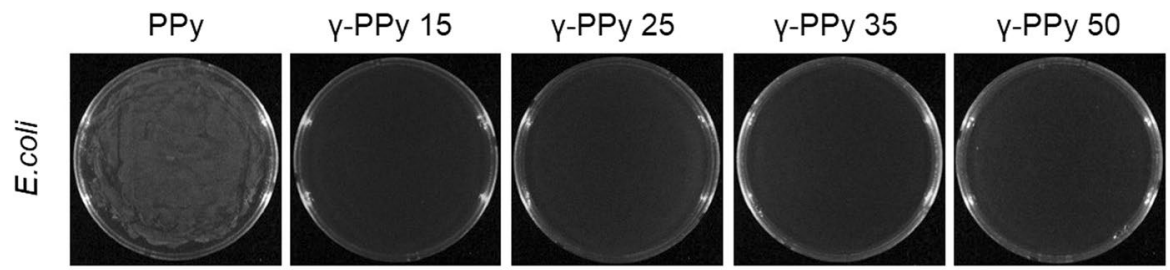

Y-PPy 75
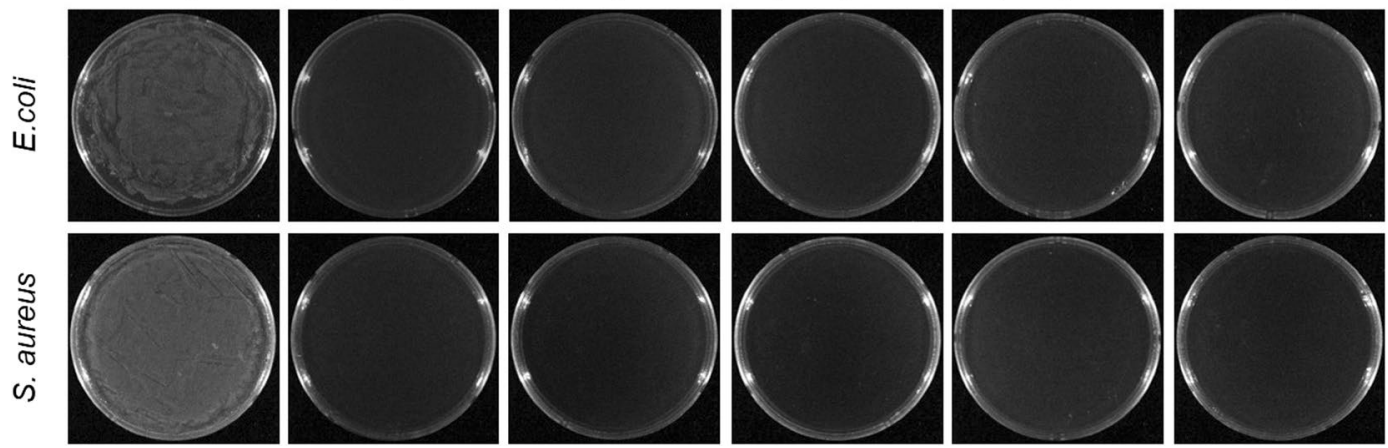

Figure 7. Sterilization test of the PPy irradiated with different doses of gamma-ray irradiation. Photographs were acquired $24 \mathrm{~h}$ after seeding the medium incubated with the irradiated PPy samples. After seeding the agar medium was incubated at $37^{\circ} \mathrm{C}$ for $18 \mathrm{~h}$.

Sterilization assay. Sterilization of PPy by gamma-ray was studied using the PPy electrodes in E. coli and $S$. aureus containing medium. After the gamma-ray irradiation $(15,25,35,50$, and $75 \mathrm{kGy})$, the irradiated PPy electrodes were further incubated in the $\mathrm{LB}$ medium at $37^{\circ} \mathrm{C}$ for $18 \mathrm{~h}$ to enrich possible bacteria that maintained their viability during the gamma-ray radiation (Supporting Information Fig. S7). As shown in Fig. 7, neither E. coli nor $S$. aureus was detected from the incubated solution. Complete bacterial eradication was observed for all the $\gamma$-PPy samples after irradiation at $15,25,35,50$, and $75 \mathrm{kGy}$. The results indicate that gamma-ray irradiation $(\geq 15 \mathrm{kGy})$ is a valid methodology for sterilization of PPy-based biomaterials.

Cytocompatibility tests of the $\gamma$-PPy. Three different cell types, including PC12 neuronal cells, C2C12 myoblasts, and NIH3T3 fibroblasts, were cultured to investigate the cytocompatibility of the various $\gamma$-PPy samples and possible changes in their cellular interactions. In particular, these cells were selected to demonstrate the potential uses of gamma-ray sterilized PPy biomaterials for various biomedical applications, such as electromyography ${ }^{34}$ and electroencephalography ${ }^{35}$, and electrically conductive scaffolds for skeletal muscle ${ }^{36}$, neu$\mathrm{ral}^{37}$, or other tissue regeneration ${ }^{38}$. As shown in Fig. 8a, cells adhered well and spread on the all of the samples (non-irradiated PPy and irradiated PPy), indicating gamma-ray irradiation at the doses of 15-75 kGy did not influence overall cellular interactions. In case of PC12 cells, the cells were differentiated by forming multiple neurites on both PPy and $\gamma$-PPy. Good adhesion, growth, and neuritogenesis of PC1 2 cells on $\gamma$-PPy were compatible with pristine PPy (non-irradiated PPy). Likewise, $\gamma$-PPy supported adhesion and growth of C2C12 and NIH3T3 
(a)

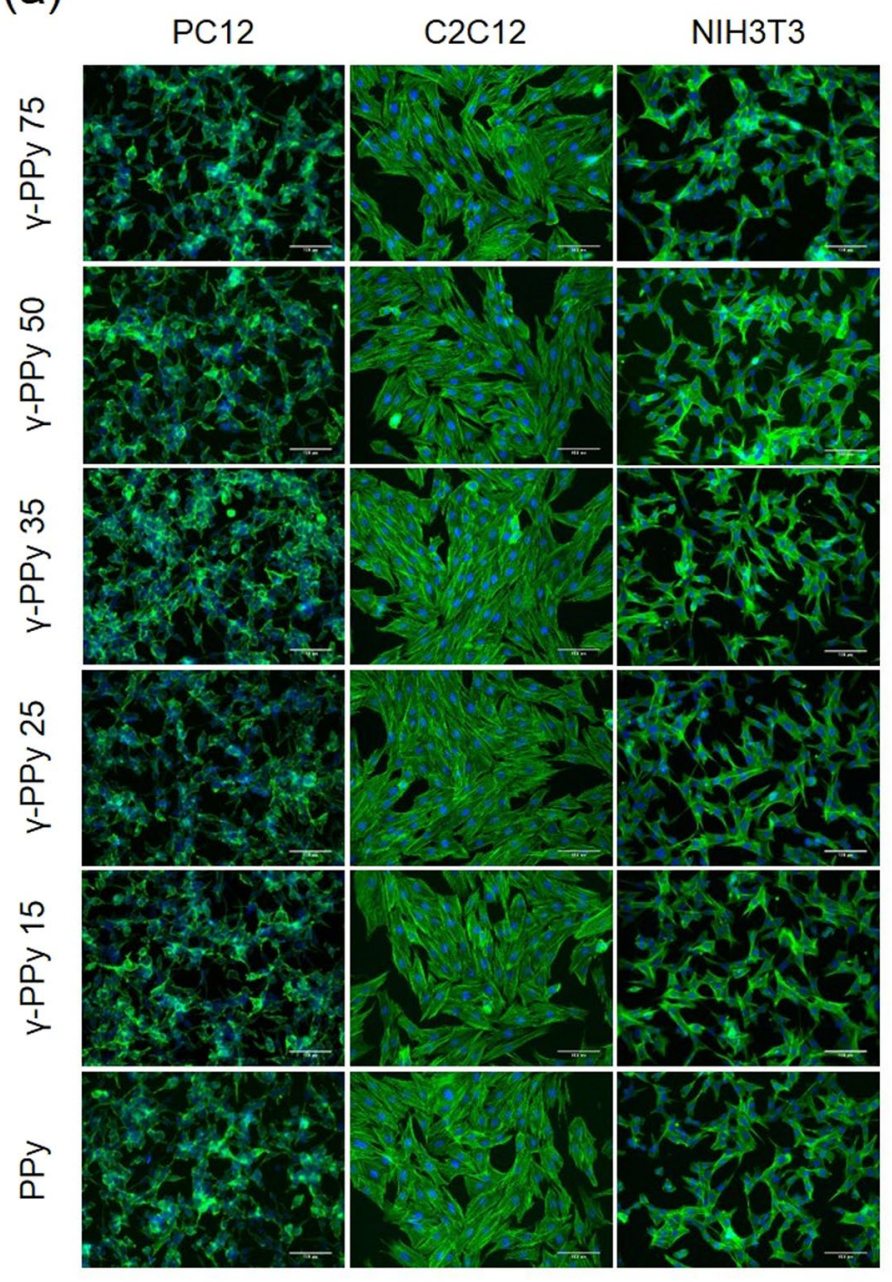

(b)
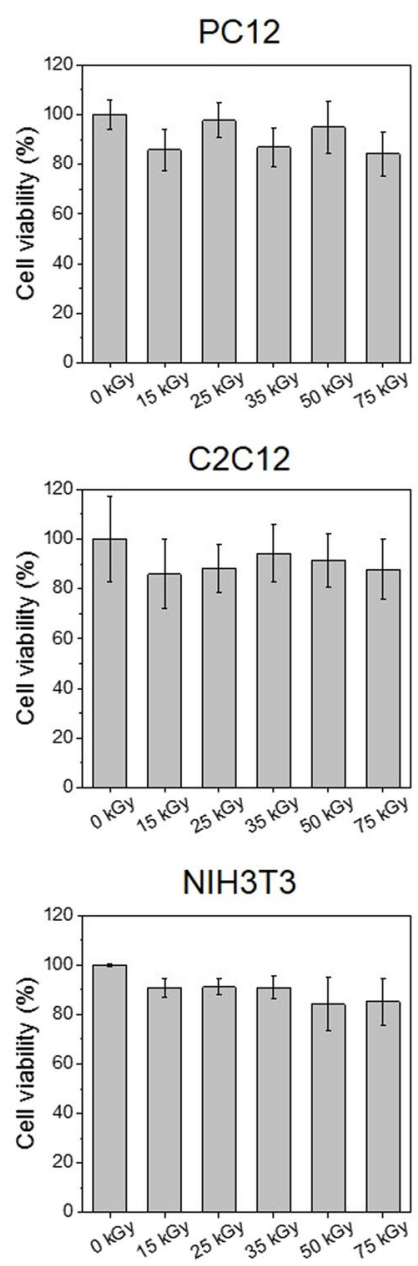

Figure 8. Cytocompatibility test of $\gamma$-PPy. (a) Immunofluoresence images of PC12, NIH3T3, and C2C12 cultured on PPy and various $\gamma$-PPy for $48 \mathrm{~h}$. Cells were stained for $F$-actin. Scale bars are $100 \mu \mathrm{m}$. (b) Viability of the various cells cultured on PPy and $\gamma$-PPy $n=3$.

cells similarly to the non-irradiated PPy. All types of cells tested in this study displayed well-spread morphologies both on the PPy and $\gamma$-PPy electrodes. WST-1 assay was performed to quantify the viability of the cells growing on various PPy and $\gamma$-PPy. As shown in Fig. 8b, the viability of the PC12 cells, C2C12 myoblasts, and NIH3T3 cell on PPy and $\gamma$-PPy was not significantly different. These results indicate that sterilization using gamma-ray irradiation does not diminish the cytocompatibility of PPy-based biomaterials (and cellular interactions with $\gamma$-PPy is similar to the pristine PPy).

\section{Discussion}

CP-based biomaterials are an emerging class of materials that efficiently mediate electrical signals with biological tissues and have a variety of potential applications ${ }^{8}$. Knowledge that electrical signals play pivotal roles in biological functions (such as electrophysiology and regeneration of damaged tissues ${ }^{39}$ ) has encouraged the development of CP-based biomaterials for various purposes such as bioelectrodes and tissue engineering scaffolds ${ }^{40}$. Bacterial infection is one of the most important issues related to the successful clinical translation of biomaterials because bacterial infections can lead to severe diseases for patients and/or malfunction of the implants ${ }^{41}$. In particular, bacterial contamination of bioelectrodes causes biofilms on the electrode surfaces, which is known as bio-fouling ${ }^{42}$. Bacterial infection and biofilm formation on electrodes does not only induce the inflammatory responses in the body but also leads to the formation of an electrically non-conductive layer on electrode surfaces, which results in the impairment of electrical and electrochemical performances of the bioelectrodes ${ }^{43}$. Therefore, sterilization of electrodes is essential for their biocompatibility and performance. To achieve manufacturing scale sterilization of biomaterials and biodevices, conventional sterilization methods such as steam, ultraviolet radiation (UV), ethylene oxide (EO), and gamma-irradiation sterilization can be employed for CP-based biomateri$\mathrm{als}^{18,19}$. The EO sterilization is widely used due to low temperature process and affordability; however, the residual toxic EO gas needs to be removed after the sterilization and thus the sterilization process requires longer time to complete the processes ${ }^{44,45}$. Steam sterilization, despite commonly employed, can cause thermal damages to 
$\mathrm{CP}$ characteristics and requires special processing to pack materials. Recently, Malliaras and colleagues studied the characteristics of PEDOT/PSS devices before and after autoclave at $121^{\circ} \mathrm{C}$ for $20 \mathrm{~min}$. They found that the PEDOT:PSS devices exhibited slightly increased impedance after the steam sterilization ${ }^{19}$. We also found that autoclave of PPy/pTS led to dramatic increases in impedance (in part attributable to cracks and local detachment of the PPy coatings), and five out of ten PPy electrodes lost their original electrochemical activities. On the other hand, sterilization by gamma-irradiation provides many advantages over steam, UV, or EO sterilization methods (e.g. simplicity and effectiveness). Our results indicate that the gamma-ray irradiation with at least $15 \mathrm{kGy}$ can eliminate the gram positive and gram negative bacteria (E. coli and S. aureus).

Irradiation with gamma-rays led to increases in hydrophilicity of PPy due to the generation of polar oxygenated carbons (i.e., $\underline{\mathrm{C}}-\mathrm{O}$ and $\underline{\mathrm{C}}=\mathrm{O}$ ) on the surface of the PPy as evident by XPS analysis. Wetting of $\mathrm{CPs}$ can play a positive role in electrochemical functions as the wetting of the CPs can benefit conductance of charge transfer at the CP surfaces ${ }^{46}$. Hydrophilic surfaces can hinder non-specific adsorption and denaturation of proteins, which can cause inflammatory reactions in vivo ${ }^{47}$. Furthermore, bonding of PPy films on electrodes was found to be greatly improved after irradiation. CP-coatings on electrode surfaces are usually not strongly adherent and often delaminated $^{48}$. It should be noted that autoclaves cause the delamination of the films and crack formation. Hence, enhanced adhesion of $\gamma$-PPy on electrodes is an important benefit to ensure in vivo performances of the PPy bioelectrodes.

We originally expected that high energy of gamma-ray could detract the electrical properties of PPy because high energy gamma-ray can cause degradation of polymer chains and intermediate molecules (e.g., oxidants) generated during the radiation might damage the chemical structures and electrical performance of the PPy-based bioelectrodes. Surprisingly, gamma-ray irradiation with the doses of 15-75 kGy did not critically influence the electrical properties of the PPy electrodes. Our conductivity measurements and UV/vis spectral analyses of the $\gamma$-PPy revealed no significant changes in the electronic states of $\gamma$-PPy, indicating minimal structural damages by PPy during gamma-ray irradiation (Fig. 5). In addition, gamma-ray irradiation of differently doped PPy (e.g., $\mathrm{PPy} / \mathrm{pTS}$, PPy/PSS, and PPy/Cl) resulted in no substantial changes in impedances, indicating that gamma-rays can be used to sterilize PPy-based biomaterials doped with various substances.

The cytocompatibility of $\gamma$-PPy was tested to ensure the sterilized PPy was cell adherent opening possibilities for applications using neuronal cells (PC12), myoblasts (C2C12), and fibroblasts (NIH3T3). The cellular morphologies and adhesion were not significantly different among PPy and $\gamma$-PPy samples, indicating the good cytocompatibility of $\gamma$-PPy. Consequently, we demonstrated that gamma-ray irradiation is suitable for sterilization of PPy-based biomaterials as their cytocompatibility and support for the adhesion and growth of various types of cells are preserved even after the gamma-ray irradiation.

\section{Conclusion}

We aimed to examine the impacts of the gamma-ray sterilization on conducting polymer (i.e., PPy)-based biomaterials. We show that PPy electrodes inoculated with E. coli and S. aureus are effectively sterilized using gamma-ray irradiation at doses of $\geq 15 \mathrm{kGy}$. The gamma-ray sterilization does not change the surface morphology of the PPy substrates, yet slight oxidation and increased hydrophilicity of PPy were observed in a dose dependent manner. Steam sterilization often caused instability of the PPy films and dramatically increased impedance; however, gamma-ray sterilization did not impair electrical and electrochemical properties of the PPy electrodes with the doses of 15-75 kGy. In vitro cell culture studies demonstrated that gamma-ray radiation did not deter cell adhesion on the substrates. We believe that gamma-ray sterilization for CP-based biomaterials can be utilized for a variety of interesting biomedical applications (including implantable electrodes, biosensors, prosthetics, and tissue engineering scaffolds).

\section{Methods}

Electrochemical fabrication of PPy/pTS electrodes. Pyrrole (Sigma-Aldrich) was purified by passing it through aluminum oxide (Sigma-Aldrich) column before use. The polymerizing solution for PPy/pTS was prepared with $0.15 \mathrm{M}$ pyrrole and $0.1 \mathrm{M}$ para-toluene sulfonate (pTS) (Sigma-Aldrich) in deionized (DI) water. For the preparation of PPy/PSS and $\mathrm{PPy} / \mathrm{Cl}$, the solutions of $0.1 \mathrm{M}$ sodium polystyrene sulfonate (NaPSS) (SigmaAldrich) and $0.1 \mathrm{M}$ sodium chloride $(\mathrm{NaCl})$ (Sigma-Aldrich) were prepared with $0.15 \mathrm{M}$ pyrrole, respectively. A PPy film was electrodeposited galvanostatically on indium-tin oxide (ITO) coated glass electrodes or gold electrodes using the three-electrode system consisting of a VersaSTAT3 electrochemical working station (Princeton Applied Research, AMEMEK Scientific Instrument, Berwyn, USA). A platinum wire electrode was used as a counter electrode, and a saturated calomel electrode (SCE, CH Instrument, Inc., TX, USA) was used as a reference electrode. The current density of $1 \mathrm{~mA} / \mathrm{cm}^{2}$ was applied to the electrolyte solution. Charges of $50 \mathrm{mC} / \mathrm{cm}^{2}$ or $100 \mathrm{mC} / \mathrm{cm}^{2}$ were employed for the electrochemical PPy deposition. The polymerized PPy films were then carefully washed with DI water.

Gamma ray irradiation of PPy electrodes. Prior to gamma-irradiation, the PPy-coated ITO electrodes were placed in a 6 well plate. The plates containing the electrodes were packed in aluminum pouches. Then, gamma 60-Co source (ACEL type C-1882) was irradiated to the samples with different radiation doses (15, 25, 35,50 , and $75 \mathrm{kGy}$ ) at a dose rate of $10 \mathrm{kGy} / \mathrm{h}$. The gamma 60-Co source was located at the Korea Atomic Energy Research Institute (KAERI), Jeongeup, Republic of Korea.

Steam sterilization (Autoclave). Steam sterilization of the PPy-deposited electrodes was performed using autoclave (Hanbaek Scientific Co., Republic of Korea). The samples were placed into autoclave pouches (Duo-Check ${ }^{\circledR}$ Sterilization Pouch, Crosstex International) and then autoclaved at $121^{\circ} \mathrm{C}$ for $20 \mathrm{~min}$. 
Surface topography analysis. The surface morphologies of PPy and $\gamma$-PPy were explored using a field emission scanning electron microscope (FE-SEM, S-4700, Hitachi, Tokyo, Japan) with an electron beam of $10 \mathrm{kV}$ and a working distance of $12.2 \mathrm{~mm}$. The samples were not coated with metals because of their conductive properties. The surface topography and roughness of the PPy and $\gamma$-PPy electrodes were analyzed by atomic force microscopy (AFM, XE-100, Park System, Seoul, Republic of Korea). The experiments were performed using an NCHR tip (NCHR Nanoworld, Neuchâtel, Switzerland). All images were acquired at a $0.3 \mathrm{~Hz}$ scan rate in tapping mode.

Water contact angle measurement. The water contact angles of the PPy and $\gamma$-PPy were measured using a Phoenix 300 contact angle analyzer (Surface Electro Optics Co., Suwon, Republic of Korea) according to the static sessile drop method. Distilled water $(10 \mu \mathrm{L})$ was dropped on three or more positions on the electrodes. Experiments were performed in triplicate.

X-ray photoelectron spectroscopy. Surface elemental analysis was performed by X-ray photoelectron spectroscopy (XPS, VG Multilab 2000 spectrometer, Thermo Scientific, Waltham, USA) equipped with an $\mathrm{Al} \mathrm{K \alpha}$ $\mathrm{X}$-ray source at $1486.6 \mathrm{eV}$ which is monochromatic. High resolution carbon $1 \mathrm{~s}\left(\mathrm{C}_{1 \mathrm{~s}}\right)$ spectra were further deconvoluted using the XPS PEAK software (University of Hong Kong, Hong Kong).

Electrochemical impedance spectroscopy (EIS). The electrochemical impedance spectra of the PPy and $\gamma$-PPy electrodes were obtained using VersaSTAT3 electrochemical working station with a three-electrode setup. Tape with a circular hole (diameter of $6 \mathrm{~mm}$ ) was attached onto the electrode surfaces to ensure the same exposed electrode areas $\left(0.2829 \mathrm{~cm}^{2}\right)$ in all samples. Impedance measurement of the electrodes was performed in the presence of redox probe solution $\left(5 \mathrm{mM}\left[\mathrm{Fe}(\mathrm{CN})_{6}\right]^{3-/ 4-}\right.$ (Sigma-Aldrich) in PBS) with an amplitude of $5 \mathrm{mV}$ in a range of $0.1-10^{5} \mathrm{~Hz}$.

Conductivity measurement. The electrical conductivity of the PPy and $\gamma$-PPy was measured using a four-point probe method (Modysystems, Korea) that the linear scan voltammetry was applied from $-1 \mathrm{~V}$ to $1 \mathrm{~V}$. The PPy film thickness was measured using a stylus profiler (Dektak XT, Bruker) and resistance was measured from the $I-V$ curve. The electrical conductivity was calculated from the film thickness and resistance according to the literature ${ }^{49}$.

Cyclic voltammetry (CV). Electrochemical properties of all of the samples were investigated by CV using a VersaSTAT3 electrochemical working station in a presence of $5 \mathrm{mM}[\mathrm{Fe}(\mathrm{CN}) 6]^{3-/ 4-}$ in $0.1 \mathrm{M} \mathrm{KCl}$ solution at a $0.1 \mathrm{~V} / \mathrm{s}$ scan rate.

UV/vis spectroscopy. PPy and $\gamma$-PPy films on ITO were analyzed by UV-vis spectroscopy (Agilent 8453, Agilent Technologies, USA). The absorbance of the irradiated bare ITO with various doses was measured and used as the backgrounds to obtain the absorbance of the $\gamma$-PPy films.

Sterilization test Escherichia coli (E. coli). (ATCC 43895) and Staphylococcus aureus (S. aureus) (ATCC 14458 ) were prepared as model gram negative and gram positive bacterium, respectively. Bacterial solutions were diluted with $1 \mathrm{~mL}$ of LB media to have a density of $1 \times 10^{7}$ for each bacterium. The PPy substrates were immersed in the bacteria-containing LB media and incubated for $24 \mathrm{~h}$ at $37^{\circ} \mathrm{C}$ with gentle shaking. Then, the media containing the electrodes was exposed to gamma-irradiation at $15,25,35,50$, and $75 \mathrm{kGy}$ with a dose rate of $10 \mathrm{kGy} / \mathrm{h}$, respectively. After the sterilization with gamma-ray, the irradiated media $(5 \mu \mathrm{L})$ was spread onto the $\mathrm{LB}$ agar plate and further by incubated for $18 \mathrm{~h}$ at $37^{\circ} \mathrm{C}$. Images of the individual plates were acquired using the ChemiDoc imaging systems (ChemiDoc, XRS+, BIO-RAD, Hercules, USA) with $0.428 \mathrm{~s}$ exposure time to check the possible colonies on the plates.

In vitro cytocompatibility test. $\mathrm{NIH} 3 \mathrm{~T} 3$ and $\mathrm{C} 2 \mathrm{C} 12$ cells were maintained in DMEM (Hyclone) containing 10\% FBS (Hyclone) and antibiotic-antimycotic solutions (Gibco) on tissue culture plate with $5 \% \mathrm{CO}_{2}$ at $37^{\circ} \mathrm{C}$. PC12 cells were maintained in DMEM medium containing $10 \%$ heat-inactivated HS (Gibco), 5\% FBS, and $1 \%$ antibiotic-antimycotic solution with $5 \% \mathrm{CO}_{2}$ at $37^{\circ} \mathrm{C}$. Culture media was exchanged every $3 \mathrm{~d}$ and cells were passaged with a $0.05 \%$ trypsin-EDTA solution (Gibco) at $90 \%$ confluence. Prior to cell culture experiments, PC12 cells were cultured on collagen coated tissue culture plate in the differentiation medium (F-12K medium (Gibco) containing $1 \%$ heat-inactivated HS, $0.5 \%$ FBS, and $1 \%$ antibiotic-antimycotic solution) with $100 \mathrm{ng} / \mathrm{mL}$ soluble nerve growth factor (NGF) (7S, murine submaxillary gland, Promega, USA) one week prior to an experiment. For cell seeding, the samples were washed three times with a deionized water and PBS (Gibco) for $1 \mathrm{~h}$. For metabolic activity measurement by the WST-1 assay, polydimethylsiloxane (PDMS) wells ( $6 \mathrm{~mm}$ in inner diameter) were placed on bare electrodes, PPy, and $\gamma$-PPy electrodes. Cells were seeded at a density of 5,000 cells into each well. The cells were incubated in the culture medium for $48 \mathrm{~h}$ with $5 \% \mathrm{CO}_{2}$ at $37^{\circ} \mathrm{C}$. After cell culture, the cell viability was quantified by the WST-1 assay according to the manufacturer's protocol. For individual sample wells, WST-1 reagent solution was added and incubated for $3 \mathrm{~h}$. Absorbance of the solution was measured at $450 \mathrm{~nm}$ using a scanning multi-well spectrophotometer (FL600, Bio-Tek, Winooski, Vermont, USA) $(n=3)$.

For immuno-staining, the cells were seeded on substrates at a density of $2 \times 10^{4} \mathrm{cells} / \mathrm{cm}^{2}$ and cultured for $48 \mathrm{~h}$. After incubation, the samples were fixed in $3.7 \%$ paraformaldehyde at room temperature for $20 \mathrm{~min}$. The samples were then washed with DPBS. Cells were permeabilized in DPBS containing $0.1 \%$ Triton X-100 for 10 min and incubated in the blocking solution (2\% bovine serum albumin (BSA) (Sigma-Aldrich) in DPBS (Gibco)) for $2 \mathrm{~min}$. For $\mathrm{F}$-actin staining, the samples were incubated at room temperature for $20 \mathrm{~min}$ in Aelxa-fluor 
488-conjugated phalloidin (Invitrogen) (1:300 in blocking solution). The samples were washed with DPBS and then incubated in 4'-6-diamidino-2-phenylindole (DAPI (Invitrogen), 1:2500 in PBS) solution to stain nuclei. Fluorescence images were acquired using florescence microscope (DMI3000B, Leica, Germany).

Statistical analysis. All tests were performed at least in triplicate and data were presented as the mean \pm standard deviation (SD) unless otherwise noted. Statistical significance was examined by one-way analysis of variance (ANOVA) with a Tukey's post-hoc comparison of the means using Origin software. A p-value less than 0.05 was considered to be statistically significant.

\section{References}

1. Guimard, N. K., Gomez, N. \& Schmidt, C. E. Conducting polymers in biomedical engineering. Prog. Polym. Sci. 32, 876-921 (2007).

2. Nambiar, S. \& Yeow, J. T. Conductive polymer-based sensors for biomedical applications. Biosens. Bioelectron. 26, 1825-1832 (2011).

3. Sanghvi, A. B., Miller, K. P.-H., Belcher, A. M. \& Schmidt, C. E. Biomaterials functionalization using a novel peptide that selectively binds to a conducting polymer. Nat. Mater. 4, 496-502 (2005).

4. Peng, H., Zhang, L., Soeller, C. \& Travas-Sejdic, J. Conducting polymers for electrochemical DNA sensing. Biomaterials 30, 2132-2148 (2009).

5. Green, R. A., Lovell, N. H., Wallace, G. G. \& Poole-Warren, L. A. Conducting polymers for neural interfaces: challenges in developing an effective long-term implant. Biomaterials 29, 3393-3399 (2008).

6. Kaur, G., Adhikari, R., Cass, P., Bown, M. \& Gunatillake, P. Electrically conductive polymers and composites for biomedical applications. RSC Adv. 5, 37553-37567 (2015).

7. Kotwal, A. \& Schmidt, C. E. Electrical stimulation alters protein adsorption and nerve cell interactions with electrically conducting biomaterials. Biomaterials 22, 1055-1064 (2001).

8. Schmidt, C. E., Shastri, V. R., Vacanti, J. P. \& Langer, R. Stimulation of neurite outgrowth using an electrically conducting polymer. Proc. Natl. Acad. Sci. 94, 8948-8953 (1997).

9. Lee, J. Y., Bashur, C. A., Goldstein, A. S. \& Schmidt, C. E. Polypyrrole-coated electrospun PLGA nanofibers for neural tissue applications. Biomaterials 30, 4325-4335 (2009).

10. Thompson, B. C. et al. Optimising the incorporation and release of a neurotrophic factor using conducting polypyrrole. J. Controlled Release 116, 285-294 (2006).

11. Richardson, R. T. et al. Polypyrrole-coated electrodes for the delivery of charge and neurotrophins to cochlear neurons. Biomaterials 30, 2614-2624 (2009).

12. Wadhwa, R., Lagenaur, C. F. \& Cui, X. T. Electrochemically controlled release of dexamethasone from conducting polymer polypyrrole coated electrode. J. Controlled Release 110, 531-541 (2006).

13. Abidian, M. R., Kim, D. \& Martin, D. C. Conducting-polymer nanotubes for controlled drug release. Adv. Mater. 18, 405-409 (2006).

14. Wang, L., Wu, Y., Hu, T., Guo, B. \& Ma, P. X. Electrospun conductive nanofibrous scaffolds for engineering cardiac tissue and 3D bioactuators. Acta Biomater. 59, 68-81 (2017).

15. Rowlands, A. S. \& Cooper-White, J. J. Directing phenotype of vascular smooth muscle cells using electrically stimulated conducting polymer. Biomaterials 29, 4510-4520 (2008).

16. Ahuja, T., Mir, I. A. \& Kumar, D. Biomolecular immobilization on conducting polymers for biosensing applications. Biomaterials 28, 791-805 (2007).

17. Kwon, O. S., Park, S. J. \& Jang, J. A high-performance VEGF aptamer functionalized polypyrrole nanotube biosensor. Biomaterials 31, 4740-4747 (2010)

18. Valente, T. et al. Effect of sterilization methods on electrospun poly (lactic acid)(PLA) fiber alignment for biomedical applications. ACS Appl. Mater. Interfaces 8, 3241-3249 (2016).

19. Uguz, I. et al. Autoclave Sterilization of PEDOT: PSS Electrophysiology Devices. Adv. Healthc. Mater. 5, 3094-3098 (2016).

20. Halpern, J. M., Gormley, C. A., Keech, M. A. \& Von Recum, H. A. Thermomechanical properties, antibiotic release, and bioactivity of a sterilized cyclodextrin drug delivery system. J. Mater. Chem. B 2, 2764-2772 (2014).

21. Costa, L. et al. Oxidation in orthopaedic UHMWPE sterilized by gamma-radiation and ethylene oxide. Biomaterials 19, 659-668 (1998).

22. Athanasiou, K. A., Agrawal, C. M., Barber, F. A. \& Burkhart, S. S. Orthopaedic applications for PLA-PGA biodegradable polymers. Arthrosc. J. Arthrosc. Relat. Surg. 14, 726-737 (1998)

23. Ali, Y., Kumar, V., Sonkawade, R., Dhaliwal, A. \& Swart, H. Gamma radiation induced modifications in Au-polypyrrole nanocomposites: Detailed Raman and X-ray studies. Vacuum 99, 265-271 (2014).

24. Ali, Y., Kumar, V., Sonkawade, R. \& Dhaliwal, A. Effect of swift heavy ion beam irradiation on Au-polyaniline composite films. Vacuum 90, 59-64 (2013).

25. Aarya, S., Dev, K., Raghuvanshi, S. K., Krishna, J. \& Wahab, M. Effect of gamma radiation on the structural and optical properties of Polyethyleneterephthalate (PET) polymer. Radiat. Phys. Chem. 81, 458-462 (2012).

26. Singh, S. \& Prasher, S. The optical, chemical and spectral response of gamma-irradiated Lexan polymeric track recorder. Radiat. Meas. 40, 50-54 (2005).

27. Khan, W., Kapoor, M. \& Kumar, N. Covalent attachment of proteins to functionalized polypyrrole-coated metallic surfaces for improved biocompatibility. Acta Biomater. 3, 541-549 (2007).

28. Stewart, E., Liu, X., Clark, G., Kapsa, R. \& Wallace, G. G. Inhibition of smooth muscle cell adhesion and proliferation on heparindoped polypyrrole. Acta Biomater. 8, 194-200 (2012).

29. Moroder, P. et al. Material properties and electrical stimulation regimens of polycaprolactone fumarate-polypyrrole scaffolds as potential conductive nerve conduits. Acta Biomater. 7, 944-953 (2011).

30. Forciniti, L., Ybarra, J., Zaman, M. H. \& Schmidt, C. E. Schwann cell response on polypyrrole substrates upon electrical stimulation. Acta Biomater. 10, 2423-2433 (2014).

31. Spearman, B. S. et al. Conductive interpenetrating networks of polypyrrole and polycaprolactone encourage electrophysiological development of cardiac cells. Acta Biomater. 28, 109-120 (2015).

32. Navale, S., Mane, A., Ghanwat, A., Mulik, A. \& Patil, V. Camphor sulfonic acid (CSA) doped polypyrrole (PPy) films: measurement of microstructural and optoelectronic properties. Measurement 50, 363-369 (2014).

33. Huyen, D. N., Tung, N. T., Vinh, T. D. \& Thien, N. D. Synergistic effects in the gas sensitivity of polypyrrole/single wall carbon nanotube composites. Sensors 12, 7965-7974 (2012).

34. Guo, L., Ma, M., Zhang, N., Langer, R. \& Anderson, D. G. Stretchable polymeric multielectrode array for conformal neural interfacing. Adv. Mater. 26, 1427-1433 (2014).

35. Leleux, P. et al. Conducting polymer electrodes for electroencephalography. Adv. Healthc. Mater. 3, 490-493 (2014).

36. Gilmore, K. J. et al. Skeletal muscle cell proliferation and differentiation on polypyrrole substrates doped with extracellular matrix components. Biomaterials 30, 5292-5304 (2009). 
37. Sudwilai, T. et al. Polypyrrole-coated electrospun poly (lactic acid) fibrous scaffold: effects of coating on electrical conductivity and neural cell growth. J. Biomater. Sci. Polym. Ed. 25, 1240-1252 (2014).

38. Zhang, J., Li, M., Kang, E.-T. \& Neoh, K. G. Electrical stimulation of adipose-derived mesenchymal stem cells in conductive scaffolds and the roles of voltage-gated ion channels. Acta Biomater. 32, 46-56 (2016).

39. Bao, R. et al. A $\pi-\pi$ conjugation-containing soft and conductive injectable polymer hydrogel highly efficiently rebuilds cardiac function after myocardial infarction. Biomaterials 122, 63-71 (2017).

40. Balint, R., Cassidy, N. J. \& Cartmell, S. H. Conductive polymers: towards a smart biomaterial for tissue engineering. Acta Biomater. 10, 2341-2353 (2014)

41. Campoccia, D., Montanaro, L. \& Arciola, C. R. A review of the biomaterials technologies for infection-resistant surfaces. Biomaterials 34, 8533-8554 (2013).

42. Singh, P. K., Parsek, M. R., Greenberg, E. P. \& Welsh, M. J. A component of innate immunity prevents bacterial biofilm development. Nature 417, 552-555 (2002).

43. Yun, Y.-H. et al. Tiny medicine: nanomaterial-based biosensors. Sensors 9, 9275-9299 (2009).

44. Mendes, G. C., Brandao, T. R. \& Silva, C. L. Ethylene oxide sterilization of medical devices: a review. Am. J. Infect. Control 35, 574-581 (2007).

45. Clough, R. High-energy radiation and polymers: A review of commercial processes and emerging applications. Nucl. Instrum. Methods Phys. Res. Sect. B Beam Interact. Mater. At. 185, 8-33 (2001).

46. Wang, J. et al. High charge/discharge rate polypyrrole films prepared by pulse current polymerization. Synth. Met. 160, 1826-1831 (2010).

47. Grainger, D. W. All charged up about implanted biomaterials. Nat. Biotechnol. 31, 507-509 (2013).

48. Ravichandran, R., Sundarrajan, S., Venugopal, J. R., Mukherjee, S. \& Ramakrishna, S. Applications of conducting polymers and their issues in biomedical engineering. J. R. Soc. Interface 7, S559-S579 (2010).

49. Fonner, J. M. et al. Biocompatibility implications for polypyrrole synthesis techniques. Biomed. Mater. 3, 034124 (2008).

\section{Acknowledgements}

This research was supported by the Basic Science Research Program (2016R1A2B4007172), Pioneer Research Center Program (2014M3C1A3001208), Global Research Laboratory (GRL) Program (2013K1A1A2A02050616) and Nuclear R\&D program (2017M2A2A6A02018535) through the National Research Foundation of Korea (NRF) funded by the Ministry of Science, ICT \& Future Planning. JGH acknowledges the support of a Lancaster University Faculty of Science and Technology "Early Career Internal Grant", a Royal Society Research Grant (RG160449), and an EPSRC Centre for Innovative Manufacturing in Large-Area Electronics Pathfinder Grant.

\section{Author Contributions}

Y.M.L. and J.L. designed the study; S.K. and J.-O.J. performed synthesis and characterization of the samples; S.L. performed spectroscopic analysis; J.-S.P., H.-J.G., and S.I.J. performed gamma-ray radiation; J.G.H. contributed to the interpretation of the data; all authors participated in development and discussions and have given approval to the final version of the manuscript.

\section{Additional Information}

Supplementary information accompanies this paper at https://doi.org/10.1038/s41598-018-22066-6.

Competing Interests: The authors declare no competing interests.

Publisher's note: Springer Nature remains neutral with regard to jurisdictional claims in published maps and institutional affiliations.

(c) Open Access This article is licensed under a Creative Commons Attribution 4.0 International (c) License, which permits use, sharing, adaptation, distribution and reproduction in any medium or format, as long as you give appropriate credit to the original author(s) and the source, provide a link to the Creative Commons license, and indicate if changes were made. The images or other third party material in this article are included in the article's Creative Commons license, unless indicated otherwise in a credit line to the material. If material is not included in the article's Creative Commons license and your intended use is not permitted by statutory regulation or exceeds the permitted use, you will need to obtain permission directly from the copyright holder. To view a copy of this license, visit http://creativecommons.org/licenses/by/4.0/.

(C) The Author(s) 2018 